\section{Integrative Medicine \\ International}

\title{
Assessment of Acupuncture and Moxibustion Effects on the Electrophysiological Properties of the Ulnar Nerve: A Nerve Conduction Study
}

\author{
Sónia Chan ${ }^{a}$ Sérgio Ferreira ${ }^{a}$ Bruno Ramos $^{a}$ Maria João Santos $^{a}$ \\ Luís Carlos Matos ${ }^{b}$ Jorge Machado ${ }^{a, c}$ \\ a ICBAS - Institute of Biomedical Sciences, University of Porto, Porto, Portugal; \\ ${ }^{b}$ Faculdade de Engenharia da Universidade do Porto, Porto, Portugal; \\ 'LABIOMEP - Biomechanics Laboratory of Porto, University of Porto, Porto, Portugal
}

\section{Keywords}

Traditional Chinese medicine - Acupuncture - Moxibustion · Nerve conduction · Ulnar nerve

\begin{abstract}
Background/Aims: Acupuncture and moxibustion, when used together, have act mechanically and thermally on treated reflexological areas. The main goal of this work was to evaluate the effects of acupuncture and moxibustion on the electrophysiological properties of the ulnar nerve. Methods: Electrical stimulation was applied to the ulnar nerve above the epicondyle of 28 volunteers. A $20-\mathrm{V}$ potential was applied, and after each 10 impulses it was increased by $10 \mathrm{~V}$, up to a maximum of $80 \mathrm{~V}$. At 20 and $80 \mathrm{~V}$, the participants were asked to rate the discomfort from 0 to 10 on a Numeric Rating Scale for pain. After the first stimulation and data collection, the control group rested for $6 \mathrm{~min}$, while the intervention group was submitted to acupuncture and moxibustion on Lingdao (HT 4). Following this period of time, a second electrical stimulation was performed on both groups. Results: The discomfort was greater in the intervention group during the second stimulation. The stimulus required to achieve the maximum amplitude decreased, but the changes were only statistically significant in the intervention group $(p=0.006)$. An increase in latency and a decrease in reaction velocity were noticed between the first and the second stimulation for both groups; however, only the control group presented statistically significant differences ( $p=0.018$ and $p=0.022$, respectively). Conclusions: Acupuncture and moxibustion on HT 4 increased the electrical sensitivity, decreased the stimulus intensity to achieve the maximum amplitude, and avoided a significant increase in latency and decrease in reaction velocity in two consecutive electrical stimulations.
\end{abstract}




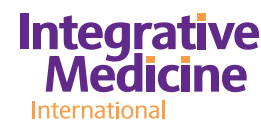

Iternational

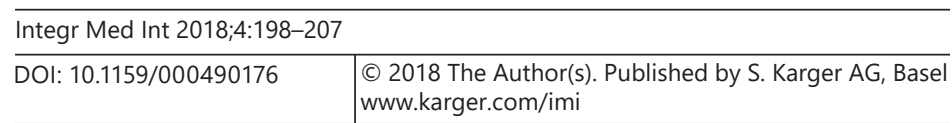

Chan et al.: Acupuncture and Moxibustion Effects on Ulnar Nerve Conduction

\section{Introduction}

Archaeological findings have shown that acupuncture was practiced in primitive societies 4,000 to 8,000 years ago, and that primitive moxibustion is related to the introduction of fire into humans' lives [1,2]. These two practices are popular and important therapeutic tools of traditional Chinese medicine (TCM), which may be understood as a system of sensations and findings designed to establish the functional vegetative state of the body $[3,4]$. TCM's therapeutic approach may include the combined or standalone use of acupuncture, moxibustion, Chinese pharmacotherapy, Chinese manual therapy, known as Tuina, and traditional biofeedback exercises such as Qigong and Tai Chi [5-10]. TCM considers the existence and relation of several elemental substances, among them qi and xue. Indeed, the concept of $q i$ is central to TCM, which posits that health is present when the $q i$ of the body is flowing smoothly and there is sufficiency but no excess [11]. Moreover, qi and xue flow through channels, also known as meridians or conduits, which are actually a connection between groups of specific points of the body surface known as acupoints [12]. According to Porkert and Hempen [13], acupuncture and moxibustion have mechanical and thermal therapeutic actions on those points. Besides the numerous clinical applications of these practices, among them those having positive effects on nerve conduction $[14,15]$, they have almost no side effects [8, 16-19].

The Heart Channel of the Hand Shaoyin is composed of interior and superficial pathways. The interior pathways originate in the heart, emerging from the system of blood vessels surrounding it, with one of the branches descending through the diaphragm to connect with the Yin-Yang-paired fu organ, the small intestine, and the other branch ascending alongside the esophagus and face to connect with the tissues surrounding the eye. The superficial pathway, on which the acupuncture points lie, travels directly from the heart to the lung and descends to emerge from the axilla at Jiquan (HT 1), then travels along the medial aspect of the upper arm (medial to the lung and pericardium channels) to the elbow at Shaohai (HT 3), along the anteromedial aspect of the lower arm to the pisiform bone of the wrist at Shenmen (HT 7), and finally through the palm and along the radial side of the little finger to terminate at the exterior corner of the nail at Shaochong (HT 9) [20, 21].

Much of the path of the heart conduit overlaps the ulnar nerve path, especially in the distal portion of the upper limb. The ulnar nerve, a major peripheral nerve of the upper limb, involved in the flexion and adduction of the hand and wrist, is one of the infraclavicular brachial plexus branches that descends down the medial aspect of the arm, posterior to the medial epicondyle, entering the forearm and innervating the majority of the intrinsic muscles and skin of the hand [22]. Nerve injuries at the elbow and wrist levels are often related to clinical conditions such as the cubital tunnel syndrome and the hand deformity known as the ulnar claw [23, 24].

The first nerve conduction studies performed in the field of basic neurobiology date back to 1870 [25]. In 1956, Gilliatt and Thomas [26] recorded the first changes in nerve conduction in patients with ulnar nerve injury. Due to the considerable volume of work on this subject [27-29], reference values for common electrophysiological parameters are well known. Nowadays, these studies not only form basic and conceptual research but also have clinical application in the diagnosis and staging of neuromuscular diseases [30-32].

A typical study on motor nerve conduction includes the stimulation of a nerve at two or more points while the compound muscle action potential is measured with a pair of electrodes [33]. Stimulation takes place by gradually increasing the electrical stimulus until the maximum response is achieved [34]. 
An electrophysiological evaluation rests on the following common parameters:

1. Latency time $\left(t_{L}\right)$, i.e., the time interval between the stimulus and the immediate response; it integrates three components: (a) the nerve activation time $\left(t_{N A}\right)$, measured between the application of the stimulus and the initiation of the action potential; (b) the nerve conduction time $\left(t_{N C}\right)$, i.e., the time the signal has spent to reach the end of the nerve after local stimulation; and (c) the neuromuscular transmission time $\left(t_{N T}\right)$, i.e., the time the signal takes to travel from the terminal axon to the motor plate, including the initiation of the muscle action potential. Thus, the latency time can be expressed as:

$T_{L}=t_{N A}+t_{N C}+t_{N T}$

2. Amplitude, i.e., the maximum distance between negative and positive peeks measured on the ordinate, relatable to the number of activated muscle fibers during nerve stimulation (excitable nerve fibers)

3. Duration, i.e., the time the signal takes to return to baseline; it evaluates the synchronization between the nerve and muscle fibers

4. Nerve conduction velocity, i.e., the signal velocity between two points of a nerve segment, assuming that the nerve activation time, the duration of neuromuscular transmission, and the generation of the action potential are constant in these two points [33-35]

The main goal of this study was to evaluate the effect of acupuncture and moxibustion on the electrophysiological properties of the ulnar nerve. For that purpose, both techniques were used simultaneously on Lingdao (HT 4), and, afterwards, nerve conduction was quantitatively accessed by the use of an electrophysiological device.

\section{Materials and Methods}

Acupuncture and moxibustion were performed by a qualified TCM practitioner. Sterile and disposable Tewa acupuncture needles of $25 \mathrm{~mm}$ length and $0.25 \mathrm{~mm}$ diameter were used in this study. The skin was disinfected with an antiseptic alcohol solution before the insertion of each needle. The moxibustion technique was performed with small moxa cylinders placed around the inserted needles. A Biopac System Pro, composed of hardware, electrodes, and the respective connecting cables, was used to induce the electrical stimulation. To reduce skin resistance, a gel was applied at the stimulation point and the electrodes were fixed with adhesive tape. The Biopac Student Lab Pro 3.7.3 software was used to acquire the electrophysiological data.

The sample was composed of 28 healthy volunteers (10 males and 18 females) aged between 20 and 35 years. After having signed an informed consent form, the volunteers were randomly assigned to two groups: a control and an intervention group, each one with 14 participants.

The electrical stimulus was applied to the supracondylar portion of the ulnar nerve of each subject's right upper limb (Fig. 1) [adapted from 36]. A 20-V stimulus was initially applied, and after each set of 10 stimuli, the voltage was increased by $10 \mathrm{~V}$ to a maximum of $80 \mathrm{~V}$. At 20 and $80 \mathrm{~V}$, the subjects were asked to rate the degree of discomfort caused by the electrical stimulation on a Numeric Rating Scale (NRS) from 0 to 10. The NRS for pain is a unidimensional measure of pain intensity for adults. It is a segmented numeric version of the visual analog scale in which the subject selects a whole number, from 0 to 10 , that best reflects the pain intensity, with 0 representing no pain and 10 extreme pain [37]. After the first electrical stimulation, the control group rested for $6 \mathrm{~min}$, while the experimental group was subjected to acupuncture and moxibustion for 6 min on Lingdao (HT 4), an acupoint from the heart conduit, also known as via vis structivae (C 4) according to the Heidelberg Model of TCM. 
Fig. 1. Photograph of the electrical stimulus being applied to the supracondylar portion of the ulnar nerve [adapted from 36].
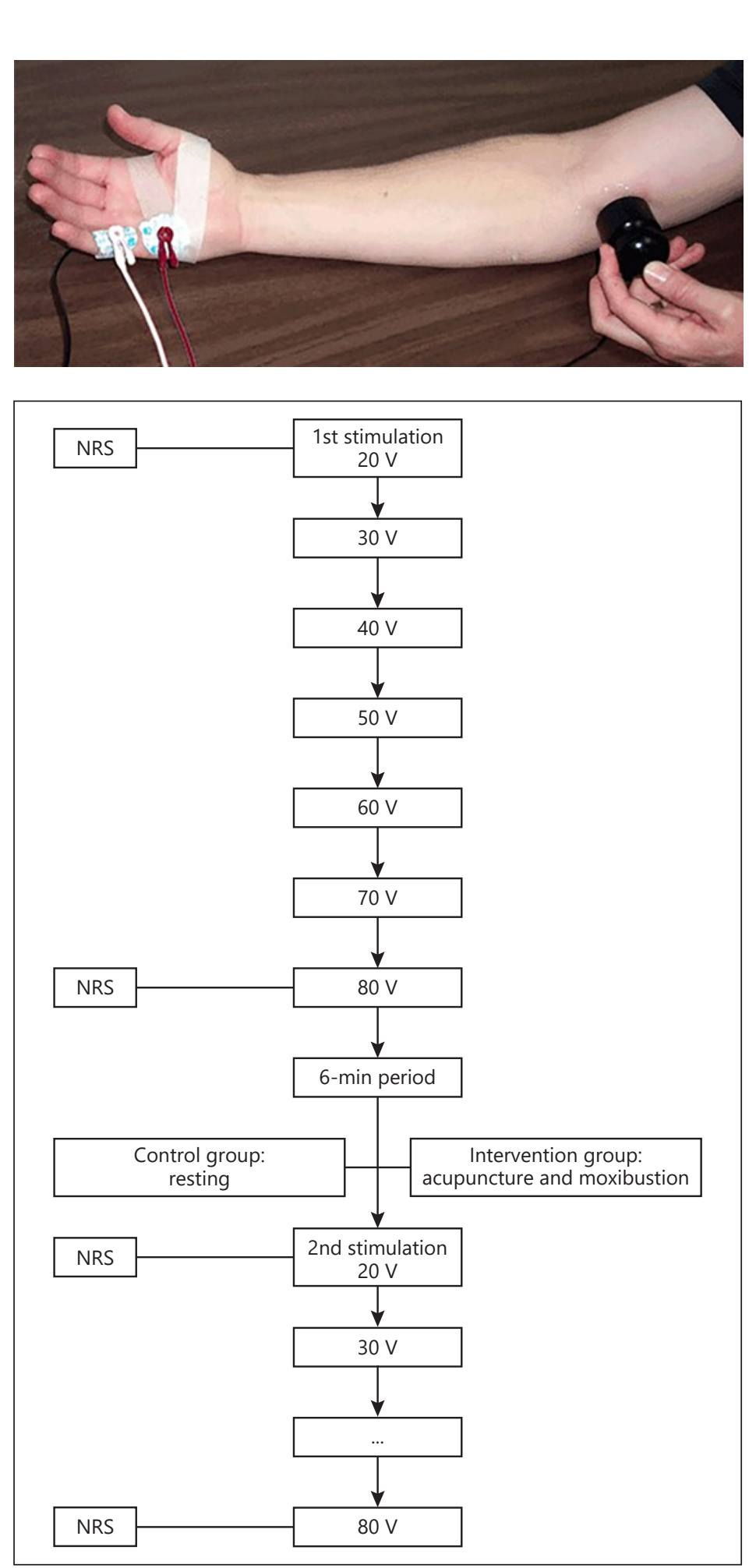

Fig. 2. Electrical stimulation methodology. NRS, Numeric Rating Scale for pain.

After this period of time, both groups repeated the previously described process. The electrical stimulation methodology is schematized in Figure 2.

Lingdao, Tongli (HT 5), Yinxi (HT 6), and Shenmen (HT 7) are four acupoints near each other, located on the radial side of the tendon of the flexor carpi ulnaris. Each one of these acupoints would be eligible for this study, but Lingdao was considered the best choice to place 
the active electrode. This choice was based not only on the anatomical proximities to the ulnar nerve and electrical stimulation point but also on its properties according to TCM theory. In keeping with the TCM view, this point is a Jing (river) Metal point - or in the Heidelberg Model terminology, a transitory acupoint, a point of transition or transport normally used when a pathogenic agent blocks the flow of $q i$ [5]. Therefore, it opens the channel promoting the $q i$ flow, acting distantly on the course of the conduit, compensating any depletion of the renal orb and dispelling external agents like ventus (wind pathogenic agent) and humor (dampness). This acupoint, also known as the Spirit Path, is used to nourish the heart and calm the "spirit" (shen) in clinical scenarios similar to hysterical neurosis, often accompanied by loss of voice $[20,21]$.

The electrophysiological parameters considered in this study were: electrical sensitivity, amplitude, latency, and reaction velocity. Considering that the branches of a terminal axon in a distal segment of a nerve are not myelinated - thus lowering the conduction of the electrical signal - and that acupuncture and moxibustion can, hypothetically, affect nerve conduction by changing the latency time, it can no longer be assumed that these parameters remain constant. In order to overcome this problem, the research team considered reaction velocity instead of conduction velocity. For this purpose, the reaction velocity $\left(v_{R}\right)$ was expressed as follows:

$$
v_{R}=L / T_{L},
$$

where $L$ is the length measured from the stimulated point to the active electrode.

\section{Statistical Analysis}

Due to the size of our sample $(n<30)$, all variables were tested for normality with the Kolmogorov-Smirnov test. With a confidence interval of 95\%, the cumulative and hypothesized distributions were compared, and if the differences were statistically significant $(p<$ $0.05)$, the variables were considered not to be normally distributed. In those cases, nonparametric methods were used. For both groups, parametric variables were tested for statistical differences with the $t$ test for independent samples. A group's sensitivity to electrical stimulation was analyzed with the Mann-Whitney test. Statistical analysis was performed with SPSS software version 22 and the results are presented as histograms elaborated in Microsoft Excel.

\section{Results}

Although the differences between the control and the intervention group were not statistically significant, all subjects reported feeling the electrical stimulus during the initial $20-V$ stimulation, except in the control group during the first stimulation. The sensitivity was assessed by using the NRS for pain, ranging from 0 to 10 , with 0 describing no discomfort and 10 the greatest discomfort. This evaluation was made by all subjects during the 20 - and $80-\mathrm{V}$ stimulations. During the $80-\mathrm{V}$ stimulation, both the control and the intervention group presented statistically significant differences between the first and second stimulations, with the average score increasing from 5.64 to $6.93(p=0.005)$ in the control group, and from 5.5 to $6.93(p=0.002)$ after acupuncture and moxibustion. As shown, the increase in sensitivity was more considerable in the intervention group (26\%) than in the control group $(22.8 \%)$.

None of the subjects presented a compound muscle action potential during the $20-\mathrm{V}$ stimulation. Actually, this potential was only detected during the $40-\mathrm{V}$ stimulation in almost all the volunteers. Moreover, the intensity of the required stimulus to generate the action 


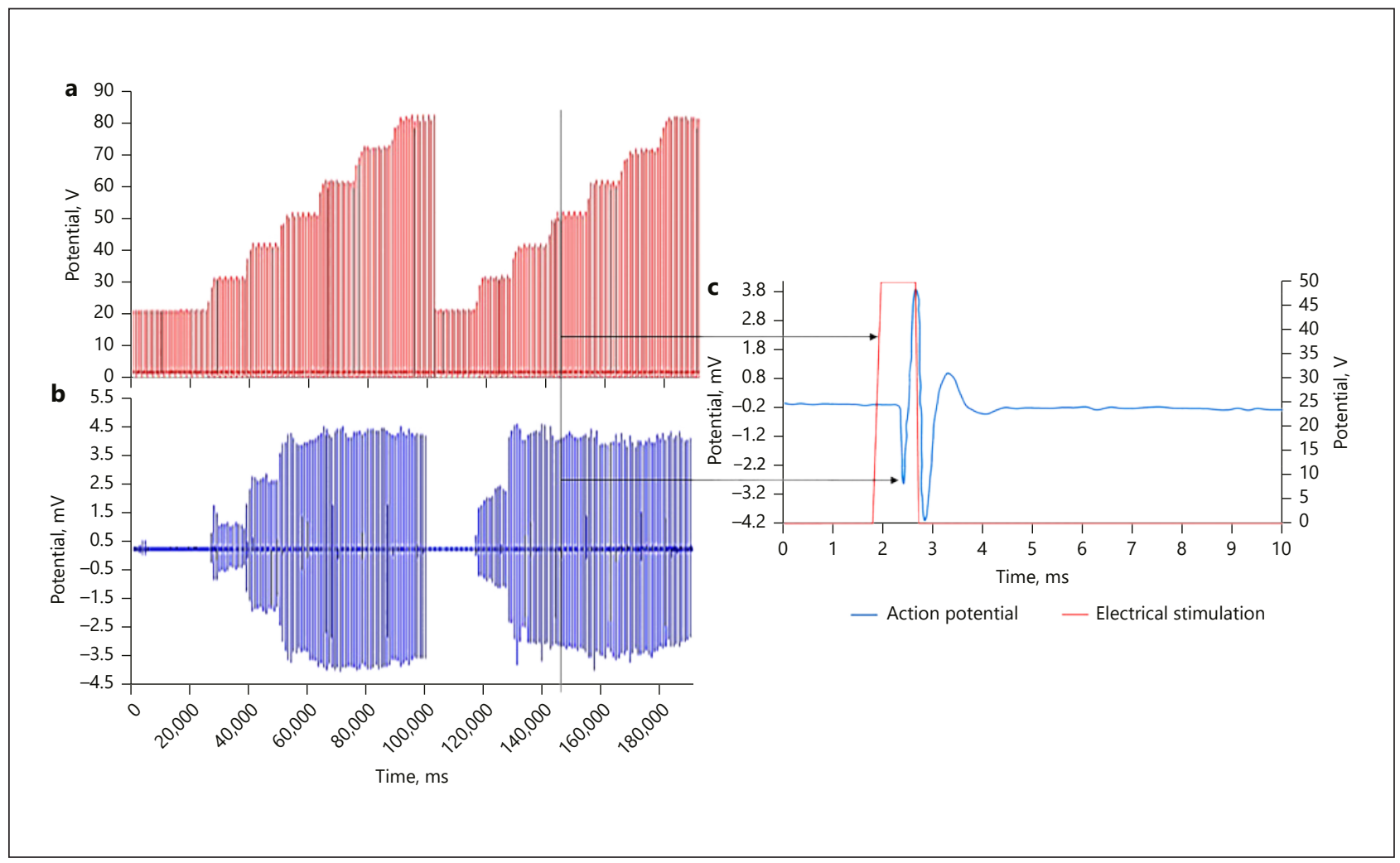

Fig. 3. Example of Biopac output data for one subject (stimulations 1 and 2). a Stimulation from 20 to $80 \mathrm{~V}$. b Action potential generated with each stimulus. c Stimulation and action potential amplification.

potential tended to be lower during the second stimulation; however, this difference was not statistically significant in either group.

Amplitude analysis allowed an indirect evaluation of the functional axons on the studied nerve. Our results show that the greater the applied stimulus, the greater the amplitude of the compound muscle action potential. As shown in Figure 3, the increase in amplitude as a function of the applied stimulus was gradual.

At a certain voltage, the amplitude reached its maximum value, remaining constant even with more intense stimulation. It was assumed that at this stage all the muscle fibers were recruited. Also, the electrical stimulus required to achieve the maximum amplitude was always smaller in the second stimulation. This decrease in intensity was only statistically significant in the intervention group ( $p=0.006)$ (Fig. 4). Comparing the maximum amplitudes achieved in the first and second stimulations, no differences were noticed in either group.

Regarding latency time, an increase was noticed from the first to the second stimulation in both groups (Fig. 5); however, the differences were only statistically significant in the control group ( $p=0.018)$.

As shown in Figure 6, both groups presented a decrease in reaction velocity during the second stimulation, but the observed differences were only statistically significant in the control group $(p=0.022)$. 
Integrative

Medicine

International

Fig. 4. Required voltage to achieve the maximum amplitude in the intervention and control groups during the 1st and 2nd stimulations.

Fig. 5. Latency time in the intervention and control groups during the 1 st and 2 nd stimulations.

Fig. 6. Reaction velocity in the intervention and control groups during the 1 st and 2 nd stimulations.

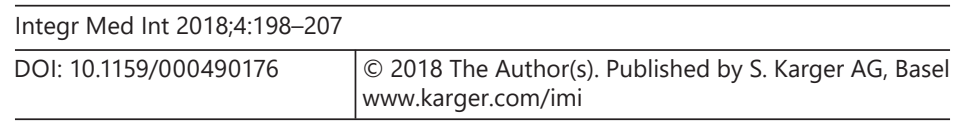

Chan et al.: Acupuncture and Moxibustion Effects on Ulnar Nerve Conduction
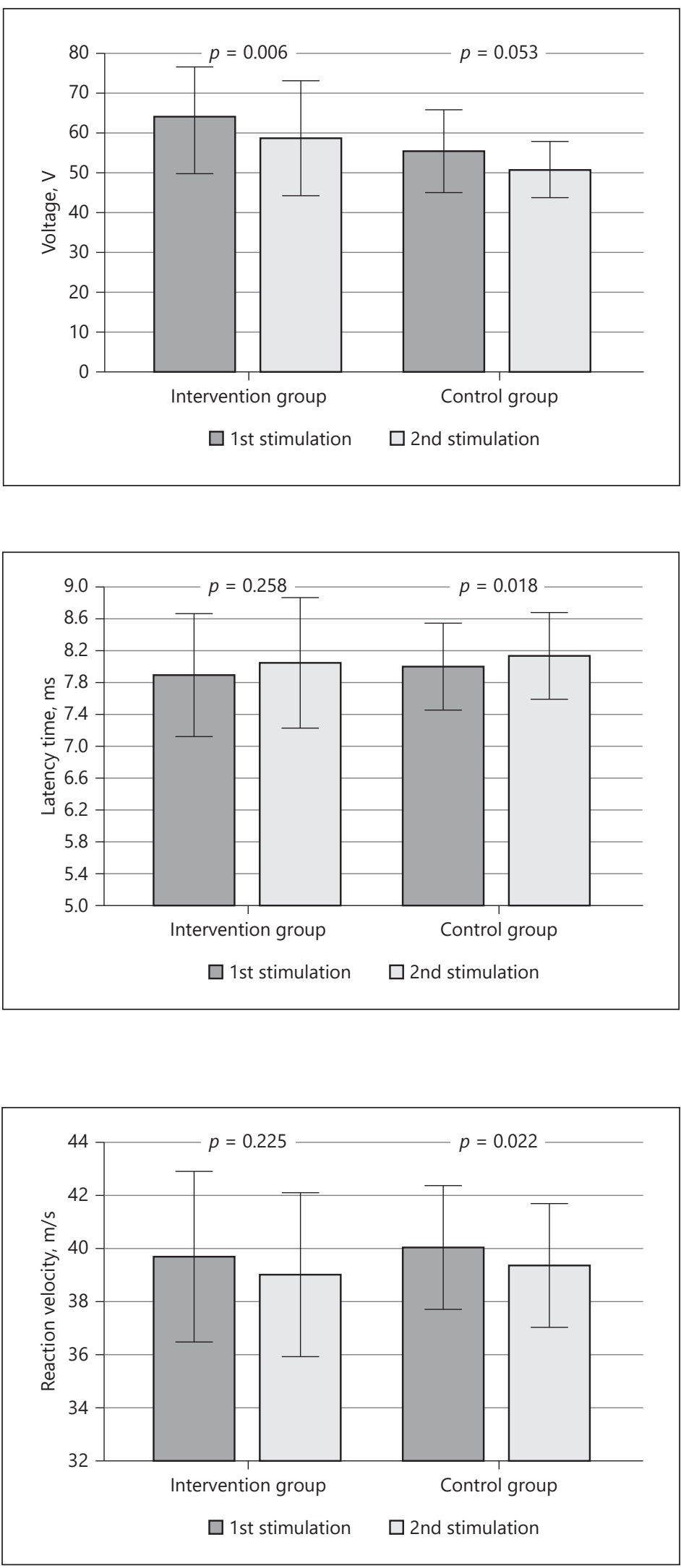


\section{Discussion}

Discomfort was evaluated during 20- and $80-\mathrm{V}$ stimulations. Although both groups demonstrated a statistically significant increase in discomfort during the $80-\mathrm{V}$ stimulation, this change was more considerable in the intervention group, suggesting that acupuncture and moxibustion may increase electrical sensitivity. Indeed, acupoints are unique locations on the body surface at which a designated electrical sensitivity is noticed and the skin's electrical conductivity is maximal compared with neighboring spots $[11,38]$. Research has shown that acupoints contain a higher density of free nerve endings, a high concentration of sensory receptors (e.g., Meissner's corpuscle, Merkel's disk, and Pacinian corpuscle) for touch and pressure, and a large number of small vessels and specialized cells such as mast cells [11]. These structural particularities might be involved in the electrophysiological response under specific stimulation. Moreover, when acupoints are coincident with or located nearby peripheral nerves, they are commonly used successfully in the treatment of peripheral neuropathy, suggesting that acupuncture induces vegetative changes in the tissue and, thereby, improvements in the electrical properties of the affected nerves $[14,15$, 39-44].

In our study, the first compound muscle action potential was detected during $40-\mathrm{V}$ stimulation in the majority of subjects. Since the voltage was increased in $10-\mathrm{V}$ steps, the action potential might have been triggered by any signal between 31 and $40 \mathrm{~V}$. The stimulus required to achieve the maximum amplitude was significantly lower during the second stimulation in the intervention group, suggesting that acupuncture and moxibustion promotes the complete recruitment of muscle fibers with a lower stimulus.

After the second stimulation, the control group presented a statistically significant increase in latency time and a decrease in reaction velocity, whereas in the intervention group - even though showing the same tendency - the differences were not statistically significant. This suggests that acupuncture and moxibustion have a positive effect on the ulnar nerve by avoiding a significant increase in latency and decrease in reaction velocity. Actually, research has shown that temperature has a marked effect on latency and conduction velocity, pointing to a decrease and an increase in these parameters, respectively, as the temperature rises [45-47]. Once moxibustion has a thermal effect on the tissue, it might condition the nerve's physiological response. In addition, acupuncture, as a vegetative activation promoter, may increase blood perfusion into the tissue, thus acting in the same way as moxibustion on these electrophysiological parameters [14].

We are aware of the limitations of this study, as the sample had a small size and the inclusion criterion was based on the participants' statement of being in good health. Moreover, a questionnaire with socioeconomic data such as the subjects' profession (for assessment of the risk of contact with hazardous materials), personal medical history (trauma, surgical history, or chronic conditions such as diabetes mellitus), or medication and sports activities (top athletes are at an increased risk of subclinical lesions [48]) should have been administered at the beginning of the study and completed by all the participants. Since the latency time depends on the elbow flexion angle, future studies should pay special attention to the uniformity of the upper limb position during data collection. As shown in other studies, the more bent the elbow, the smaller the conduction velocity [27, 49]. Despite the mentioned limitations, this prospective study has met the intended objectives and presents promising results for larger-scale follow-up studies. 


\section{Conclusion}

As an overall conclusion, it can be stated that stimulation of Lingdao (HT 4) by acupuncture and moxibustion has an electrophysiological effect on ulnar nerve conduction by (a) decreasing the intensity of the required stimulus to achieve the maximum amplitude (i.e., less stimulation is needed for the full recruitment of muscle fibers) and (b) avoiding a significant increase in latency time and, consequently, a decrease in reaction velocity.

\section{Acknowledgements}

We are grateful to all the volunteers that kindly accepted to participate in this study.

\section{Disclosure Statement}

The authors declare no conflicts of interest.

\section{References}

1 Greten HJ: Understanding TCM: Scientific Chinese Medicine - The Heidelberg Model. Heidelberg, Heidelberg School Editions, 2008.

2 Run-Ming Y: The origin and development of Chinese acupuncture and moxibustion. Anc Sci Life 1985;4:224228.

3 Greten HJ: Chinese medicine as vegetative systems biology. Part I: therapeutic methods (in German). HNO 2011;59:1160-1164.

4 Greten HJ: Chinese medicine as vegetative systems biology. Part II: the structure of TCM diagnosis (in German). HNO 2011:59:1165-1175.

5 Greten HJ: Understanding TCM: The Fundamentals of Chinese Medicine. Part I. Heidelberg, Heidelberg School Editions, 2013.

6 Greten HJ: Understanding TCM: The Fundamentals of Chinese Medicine. Part II. Heidelberg, Heidelberg School Editions, 2014.

7 Matos LC, Sousa CM, Gonçalves M, Gabriel J, Machado J, Greten HJ: Qigong as a traditional vegetative biofeedback therapy: long-term conditioning of physiological mind-body effects. Biomed Res Int 2015;2015:531789.

8 Teixeira J, Santos MJ, Matos LC, Machado JP: Evaluation of the effectiveness of acupuncture in the treatment of knee osteoarthritis: a case study. Medicines (Basel) 2018;5:E18.

9 Rodrigues JMS, Mestre MICP, Matos LC, Machado JP: Effects of taijiquan and qigong practice over behavioural disorders in school-age children: a pilot study. J Bodyw Mov Ther, in press.

10 Matos L, Goncalves MJ, Silva AR, Mendes JG, Machado JP, Greten HJ: Assessment of Qigong-related effects by infrared thermography=a case study. Zhong Xi Yi Jie He Xue Bao 2012;10:663-666.

11 Cassidy CM: Contemporary Chinese Medicine and Acupuncture. New York, Churchill Livingstone, 2002.

12 Wang G-J, Ayati MH, Zhang W-B: Meridian studies in China: a systematic review. J Acupunct Meridian Stud 2010;3:1-9.

13 Porkert M, Hempen C-H: Classical Acupuncture: The Standard Textbook. Dinkelscherben, Phainon Ed and Media, Acta Medicinae Sinensis, 1995.

14 Schröder S, Liepert J, Remppis A, Greten JH: Acupuncture treatment improves nerve conduction in peripheral neuropathy. Eur J Neurol 2007;14:276-281.

15 Schröder S, Remppis A, Greten T, Brazkiewicz F, Morcos M, Greten HJ: Quantification of acupuncture effects on peripheral neuropathy of unknown and diabetic cause by nerve conduction studies. J Acupunct Tuina Sci 2010;6:312-314.

16 Witt CM, Pach D, Brinkhaus B, Wruck K, Tag B, Mank S, et al: Safety of acupuncture: results of a prospective observational study with 229,230 patients and introduction of a medical information and consent form. Forsch Komplementmed 2009;16:91-97.

17 WHO: Acupuncture: Review and Analysis of Reports on Controlled Clinical Trials. Geneva, World Health Organization, 2002.

18 Zhang ZJ, Chen HY, Yip KC, Ng R, Wong VT: The effectiveness and safety of acupuncture therapy in depressive disorders: systematic review and meta-analysis. J Affect Disord 2010;124:9-21. 
19 Yue J: Treatment of 78 patients with knee osteoarthritis by acupuncture, moxibustion, and tuina. World J Acupunct Moxibustion 2015;25:53-56.

20 Deadman P, Al-Khafaji M, Baker K: A Manual of Acupuncture. Hove, Journal of Chinese Medicine Publications, 1998.

21 Focks C: Atlas of Acupuncture. New York, Churchill Livingstone Elsevier, 2008.

22 Netter FH: Atlas of Human Anatomy. Philadelphia, Elsevier Health Sciences, 2017.

23 Staples JR, Calfee R: Cubital tunnel syndrome: current concepts. J Am Acad Orthop Surg 2017;25:e215-e224.

24 Taylor NL, Raj AD, Dick HM, Solomon S: The correction of ulnar claw fingers: a follow-up study comparing the extensor-to-flexor with the palmaris longus 4-tailed tendon transfer in patients with leprosy. J Hand Surg 2004;29:595-604.

25 Turner MR, Kiernan MC: Landmark Papers in Neurology. New York, Oxford University Press, 2015.

26 Gilliatt RW, Thomas PK: Changes in nerve conduction with ulnar lesions at the elbow. J Neurol Neurosurg Psychiatry 1960;23:312-320.

27 Albers J: Nerve Conduction Manual. Ann Arbor, Electroneuromyography Laboratory, Department of Physical Medicine and Rehabilitation, University of Michigan Hospital, Ann Arbor, 2017.

28 Anton D, Gerr F, Merlino L, Rosecrance JC: Normative median and ulnar nerve conduction values among a rural aged population. Work 2014;49:5-14.

29 Thomas PK, Fullerton PM: Nerve fibre size in the carpal tunnel syndrome. J Neurol Neurosurg Psychiatry 1963; 26:520-527.

30 Ginanneschi F, Milani P, Rossi A: Anomalies of ulnar nerve conduction in different carpal tunnel syndrome stages. Muscle Nerve 2008;38:1155-1160.

31 Mallik A, Weir A: Nerve conduction studies: essentials and pitfalls in practice. J Neurol Neurosurg Psychiatry 2005;76(suppl 2):ii23-ii31.

32 Kasper D, Fauci A, Hauser S, Longo D, Jameson JL, Loscalzo J: Harrison’s Principles of Internal Medicine, 19e. McGraw-Hill, 2015.

33 Kim DH: Ulnar nerve conduction study of the first dorsal interosseous muscle in Korean subjects. Ann Rehabil Med 2011;35:658-663.

34 Kimura J: Electrodiagnosis in Diseases of Nerve and Muscle: Principles and Practice. New York, Oxford University Press, 2001

35 Oh SJ: Clinical Electromyography: Nerve Conduction Studies. Philadelphia, Lippincott Williams \& Wilkins, 2003.

36 Biopac Systems, I. H03 Nerve Conduction-MP36/35. 2018. https://www.biopac.com/curriculum/h03-nerveconduction-mp3635/.

37 Hawker GA, Mian S, Kendzerska T, French M: Measures of adult pain: Visual Analog Scale for Pain (VAS Pain), Numeric Rating Scale for Pain (NRS Pain), McGill Pain Questionnaire (MPQ), Short-Form McGill Pain Questionnaire (SF-MPQ), Chronic Pain Grade Scale (CPGS), Short Form-36 Bodily Pain Scale (SF-36 BPS), and Measure of Intermittent and Constant Osteoarthritis Pain (ICOAP). Arthritis Care Res (Hoboken) 2011;63(suppl 11):S240-S252.

38 Xu J: The Praeger Handbook of Acupuncture for Pain Management: A Guide to How the "Magic Needles" Work. Santa Barbara, ABC-CLIO, 2014.

39 Tong Y, Guo H, Han B: Fifteen-day acupuncture treatment relieves diabetic peripheral neuropathy. J Acupunct Meridian Stud 2010;3:95-103.

40 Dimitrova A, Murchison C, Oken B: Acupuncture for the treatment of peripheral neuropathy: a systematic review and meta-analysis. J Altern Complement Med 2017;23:164-179.

41 Khosrawi S, Moghtaderi A, Haghighat S: Acupuncture in treatment of carpal tunnel syndrome: a randomized controlled trial study. J Res Med Sci 2012;17:1-7.

42 Dimitrova A: Introducing a standardized acupuncture protocol for peripheral neuropathy: a case series. Med Acupunct 2017;29:352-365.

43 Ural FG, Öztürk GT: The acupuncture effect on median nerve morphology in patients with carpal tunnel syndrome: an ultrasonographic study. Evid Based Complement Alternat Med 2017;2017:7420648.

44 Cox J, Varatharajan S, Côté P; Optima Collaboration: Effectiveness of acupuncture therapies to manage musculoskeletal disorders of the extremities: a systematic review. J Orthop Sports Phys Ther 2016;46:409-429.

45 Todnem K, Knudsen G, Riise T, Nyland H, Aarli JA: The non-linear relationship between nerve conduction velocity and skin temperature. J Neurol Neurosurg Psychiatry 1989;52:497-501.

46 Dhavalikar M, Narkeesh A, Gupta N: Effect of skin temperature on nerve conduction velocity and reliability of temperature correction formula in Indian females. J Exerc Sci Physiother 2009;5:24-29.

47 Herrera E, Sandoval MC, Camargo DM, Salvini TF: Motor and sensory nerve conduction are affected differently by ice pack, ice massage, and cold water immersion. Phys Ther 2010;90:581-591.

48 Singh S, Kaur S: Study of motor nerve conduction velocities of upper extremity in the female archers. Int J Phys Educ Sports Health 2015;1:31-33.

49 Sattari S, Emad M: Changes in ulnar nerve conduction velocity across different angles of elbow flexion. Electromyogr Clin Neurophysiol 2007;47:373-376. 